\title{
Fungos associados às sementes (Cariopses) de cana-de-açúcar: métodos para detecção, incidência e relação entre incidência fúngica e ambiente de produção das sementes.
}

\author{
Thais Dias Martins ${ }^{1,3}$; José Otavio Machado Menten ${ }^{1}$; Álvaro Sanguino²
}

\begin{abstract}
${ }^{1}$ Departamento de Entomologia, Fitopatologia e Zoologia Agrícola, Escola Superior de Agricultura Luiz de Queiroz, Av. Pádua Dias, 11, 13418900, Piracicaba, SP; ${ }^{2}$ Centro de Tecnologia Canavieira/ Fazenda Sto. Antônio, CP 162, 13400-000, Piracicaba, SP; ${ }^{3}$ Bolsista CNPq Parte da dissertação do primeiro autor, apresentada à ESALQ/ USP.

Autor para correspondência: José Otavio Machado Menten. (jomenten@esalq.usp.br)

Data de chegada: 01/12/2006. Aceito para publicação em: 17/12/2008
\end{abstract}

\section{RESUMO}

Martins, T.D.; Menten, J.O.M.; Sanguino, A.. Fungos associados às sementes (Cariopses) de cana-de-açúcar: métodos para detecção, incidência e relação entre incidência fúngica e ambiente de produção das sementes. Summa Phytopathologica, v.35, n.3, p.173-178, 2009

O presente trabalho teve como objetivos: determinar o método mais adequado para detecção e identificação de fungos associados às sementes (cariopses) de cana-de-açúcar; caracterizar os fungos associados; verificar as incidências nessas sementes e relacionar a incidência fúngica nessas sementes com o ambiente onde foram produzidas. Para detecção do método mais adequado, foram comparados dois substratos, em placas de Petri: agar-água com papel quadriculado e papel de filtro. Utilizaram-se placas de Petri de plástico e de vidro do tipo pirex, para verificar a influência do recipiente. Também foram comparados dois regimes de luz ( $12 \mathrm{~h}$ luz branca fluorescente/12 h escuro e escuro contínuo). As sementes foram mantidas durante sete dias sob temperatura constante de $28 \pm 2^{\circ} \mathrm{C}$, quando se procedeu à avaliação. Os requisitos para comparação dos métodos foram sensibilidade, economicidade e praticidade. A partir do método determinado como o mais adequado, foi realizada análise sanitária de 29 cruzamentos dos anos de 2002, 2003 e 2004 caracterizando os fungos associados e verificando as incidências Posteriormente, compararam-se estas incidências com as condições ambientes, de temperatura e umidade relativa, em que as sementes foram produzidas no programa de melhoramento genético. O método considerado mais adequado, de acordo com os parâmetros analisados, foi o do papel de filtro em placa de Petri de plástico e incubação sob regime de luz ( $12 \mathrm{~h}$ luz branca fluorescente/12 h escuro). Os fungos detectados foram: Alternaria alternata; Aspergillus sp.; Bipolaris sacchari; três grupos morfológicos distintos pertencentes ao gênero Bipolaris; dois grupos morfológicos de Cladosporium; Colletotrichum sp.; três grupos morfológicos de Curvularia; Epicoccum sp.; Fusarium verticillioides; Fusarium semitectum; Leptosphaerulina sp.; Nigrospora sp.; Penicillium sp.; Periconia sp.; Phoma herbarum; Rhizopus sp. e Trichoderma sp. Os mais freqüentemente encontrados foram: Bipolaris sacchari; Bipolaris spp.; Cladosporium spp.; Curvularia spp.; Fusarium verticillioides; Fusarium semitectum e Phoma herbarum. Quando se comparou a porcentagem de incidência dos diversos fungos com o ambiente de produção das sementes, observou-se que não houve relação de temperatura e umidade relativa com incidência fúngica. Supõe-se que as variações de incidência possam estar relacionadas com diferentes fontes de inóculo nos locais de cruzamento ou características genéticas das sementes.

Palavras-chave adicionais: Substrato; Regime de luz; Placas de Petri; Incidência fúngica

\section{ABSTRACT}

Martins, T.D.; Menten, J.O.M.; Sanguino, A.. Sugarcane seedborne (Caryopses) fungi: detection methods, incidence and relation between fungical incidence and seed production conditions. Summa Phytopathologica, v.35, n.3, p.173-178, 2009

This study had as objectives: to determine the most adequate method for detection and identification of sugarcane seedborne fungi; to characterize the associated fungi; to verify the incidences and to relate the fungical incidence in these seeds with the conditions where they were produced. To know the most adequate method of detection, two substrates were compared in Petri dishes: filter paper on agarwater culture medium and only filter paper. Glass and plastic Petri plates were utilized, to verify the recipient influence. It was also compared two light regimens ( $12 \mathrm{~h}$ alternating cycles of light and darkness and continuous darkness). The seeds were kept during seven days under constant temperature of $28 \pm 2{ }^{\circ} \mathrm{C}$, when the evaluations were proceeded. The requirements for comparison of the methods were sensitivity, economicity and practicity. After the determination of the most adequate method, were done health tests of 29 crosses of the years 2002, 2003 and 2004, to characterize the seedborne fungi and verify their incidences. Later, these incidences were compared to the conditions of temperature and relative humidity from where the seeds were produced at the breeding program. The method considered most appropriated, in agreement with the analyzed parameters, was the filter paper in plastic Petri plate and the incubation under light regimen ( $12 \mathrm{~h}$ alternating cycles of light and darkness). The detected fungi were: Alternaria alternata; Aspergillus sp.; Bipolaris sacchari; three distinct morphologic groups (GM) of the Bipolaris genera; two morphological groups of the Cladosporium; Colletotrichum sp.; three morphological groups of Curvularia; Epicoccum sp.; Fusarium verticillioides; Fusarium semitectum; Leptosphaerulina $\mathrm{sp}$; Nigrospora sp.; Penicillium sp.; Periconia sp.; Phoma herbarum; Rhizopus sp. and Trichoderma sp. The most frequently found were: Bipolaris sacchari; Bipolaris spp.; Cladosporium spp.; Curvularia spp.; Fusarium verticillioides; Fusarium semitectum and Phoma herbarum. When the incidence percentage of the diverse fungi was compared with the seed production conditions, the relation of temperature and relative humidity with fungical incidence was not noticed. It was considered that the incidence's variations can be related to different sources of inoculum where the plants were crossed or to genetic characteristics of the seeds.

Keywords: Substract; Light regimen; Petri dishes; Fungical incidence 
A cana-de-açúcar é uma cultura de grande importância no mundo, sendo utilizada, principalmente, para obtenção de açúcar e de álcool etílico.

Um dos caminhos para aumentar o rendimento da cana-de-açúcar são os estudos que propõem métodos de melhoramento genético da cultura (6). Nesses programas, as sementes equivalem ao produto do cruzamento de duas variedades que tenham qualidades desejáveis. Há, contudo, patógenos que causam a morte dessas sementes ou plântulas, resultando na falta de materiais para futura obtenção de uma boa variedade.

As plântulas suscetíveis aos patógenos morrem depois de três ou quatro dias da germinação, segundo Byther \& Steiner (2). As plântulas resistentes, que conseguem crescer na presença dos patógenos em duas ou três semanas, podem se recuperar da infecção.

Os principais agentes causais das doenças que limitam a produção das sementes de cana-de-açúcar são os fungos que, quando associados a essas, são beneficiados pela maneira e pelas condições ambientes (altas umidade e temperatura) em que as sementes são produzidas (12). Estes patógenos causam perdas significativas em viveiros de plântulas, limitando o sucesso e a rapidez dos programas de melhoramento genético.

Loveless \& Smith (7) relataram perdas de até $50 \%$ de plântulas, na Jamaica, por um patógeno associado às sementes de cana-de-açúcar, Bipolaris sacchari. Byther \& Steiner (2) relataram a perda de $8 \%$ a $31 \%$ de plântulas de cana-de-açúcar por patógenos de sementes, na Estação Experimental da Hawaiian Sugar Planters' Association.

Segundo Sanguino (A. Centro de Tecnologia Canavieira (CTC)), a porcentagem de perdas de plântulas por fungos associados às sementes pode atingir $100 \%$ em determinados cruzamentos, caso não se empreguem medidas de controle (comunicação pessoal).

Para o controle eficiente dos patógenos prejudiciais às "sementes" verdadeiras, é necessária a realização de sua análise sanitária, com o propósito de identificar os fungos associados e definir o foco do tratamento químico.

Alguns trabalhos empregam a análise sanitária de sementes de cana-de-açúcar $(7,12,13,14)$. Há, entretanto, grande divergência sobre qual o melhor método para análise.

Sanguino (12) utilizou o método do agar-água, sob papel sulfite quadriculado, em placas de Petri e encontrou Alternaria, Cladosporium, Curvularia, Fusarium, Bipolaris, Monilia, Nigrospora, Pestalotia, Phoma, Phitomyces, Rhizopus e Trichoderma. Silva (13) utilizou o método de distribuição de sementes em meio agar-água em placas de Petri. Seus resultados foram similares aos de Sanguino (12).

Em estudo comparativo, Narendra \& Setty (10) empregaram os métodos agar-água e papel de filtro em placas de Petri e constataram que o segundo método resultou em melhores resultados do que o primeiro. Os gêneros associados às sementes, encontrados por estes autores, foram: Alternaria, Aspergillus, Cephalosporium, Chaetomium, Cladosporium, Curvularia, Diplodia, Bipolaris, Epicoccum, Fusarium, Lacellinopsis, Malustela, Memnoniella, Nigrospora, Penicillium, Phoma, Phyllosticta, Pyrenochaeta, Scopulariopsis e Trichoconis. Em muitos desses gêneros encontrados foram identificadas diversas espécies.

Outra comparação entre dois métodos de detecção de fungos em sementes de cana-de-açúcar (batata-dextrose-agar e papel de filtro em placas de Petri) foi feita por Sinha \& Singh (14). Esses pesquisadores encontraram as seguintes espécies: Alternaria alternata, Aspergillus flavus, A. fumigatus, A. niger, Chaetomium globosum, Curvularia lunata, C. verruculosa, Bipolaris hawaiiensis, B. rostrata, B. sacchari,
Fusarium moniliforme, F. oxysporum, F. semitectum, Phoma sorghina, Penicillium sp. e Rhizopus arrhizus. Os resultados obtidos na comparação dos métodos mostraram que o do papel de filtro foi o mais sensível e apresentou maior freqüência dos fungos, quando comparado ao método de batata-dextrose-agar.

Dentre as determinações dos fungos apresentadas pelos autores que estudaram a incidência de fungos associados às sementes de canade-açúcar, observa-se que existem gêneros que só foram relatados em determinados países. Um desses casos é o da Índia, país de origem da cana-de-açúcar, onde foram relatados os gêneros: Cephalosporium, Chaetomium, Diplodia, Lacellinopsis, Malustela, Memnoniella, Phylosticta, Pyrenochaeta, Scopulariopsis e Trichoconis (10). No Brasil, Sanguino (12) constatou pela primeira vez gêneros como Monilia, Pestalotia, Phytomices e Trichoderma. Outros, como Curvularia e Bipolaris, têm ocorrência generalizada e Cladosporium, Alternaria, Aspergillus, Fusarium, Phoma, Penicillium, Rhizopus e Epicoccum já foram relatados na Índia e no Brasil.

De acordo com Lucca Filho (8) e Rego (11), os métodos do papel de filtro e o plaqueamento em agar são os mais efetivos na detecção de fungos transportados por sementes, pois são capazes de revelar a presença de hifas, corpos de frutificação e esporos, independentemente da sua localização, externa ou interna nas sementes.

Além de sensibilidade na detecção, Mathur \& Kongdal (9) analisam os parâmetros de praticidade e economicidade. Segundo esses autores, o método do papel de filtro é simples e barato, além de ser útil na detecção de patógenos e outros microrganismos associados às sementes.

Considerando a diversidade de opiniões sobre os métodos para a análise sanitária da semente de cana-de-açúcar, o presente estudo teve como objetivos investigar o método mais adequado para detecção de fungos associados às sementes (cariopses) de cana-de-açúcar; caracterizar os fungos associados e verificar as suas incidências nessas sementes; e relacionar a incidência fúngica nessas sementes e o ambiente onde foram produzidas.

\section{MATERIAL E MÉTODOS}

Os experimentos foram realizados no Laboratório de Patologia de Sementes do Departamento de Entomologia, Fitopatologia e Zoologia Agrícola da ESALQ - USP e no Laboratório de Fitopatologia do Centro de Tecnologia Canavieira (CTC) em Piracicaba-SP.

As sementes eram provenientes de plantas híbridas produzidas nos programas de melhoramento realizados em 2002, 2003 e 2004, na Estação Experimental do CTC, com sede em Camamú (BA), e semibeneficiadas, retirando os pêlos e talos, restando apenas a cariopse envolta pelas pálea e lema.

Para a detecção do método mais adequado, foram comparados dois substratos, em placas de Petri: agar-água com papel quadriculado e papel de filtro. Utilizaram-se placas de Petri de plástico e de vidro do tipo pírex, para verificar a influência do recipiente. As sementes eram provenientes de três cruzamentos: SP81231 X SP963295; SP953387 X RB815396 e SP992254 X SP953163, todos de 2004.

No método do agar-água, foram distribuídas 25 sementes por placa de Petri de vidro e de plástico, com papel sulfite quadriculado ( $75 \mathrm{~g} /$ $\mathrm{m}^{2}$ ) sobre meio agar-água (para fornecer umidade), colocando-se uma semente por quadrícula do papel. E o método do papel de filtro consistiu em distribuir 25 sementes eqüidistantes em três folhas de papel de filtro umedecidos com água destilada, em placas de Petri de 


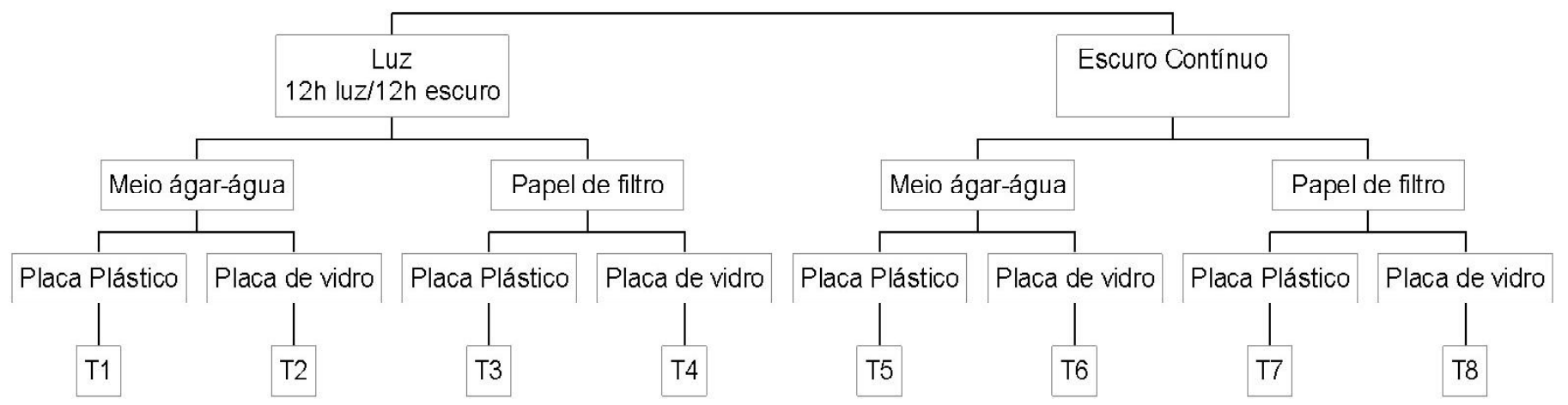

Figura 1- Organograma dos tratamentos utilizando diferentes regimes de luz, material de placa de Petri e substrato

plástico e de vidro.

Nos diferentes métodos, as placas foram mantidas sob regimes de luz alternada ( $12 \mathrm{~h}$ luz branca fluorescente/ $12 \mathrm{~h}$ escuro) e escuro contínuo, por sete dias, sob temperatura de $28 \pm 2{ }^{\circ} \mathrm{C}$.

As avaliações foram realizadas examinando-se todas as sementes sob estereomicroscópio e, se necessário, microscópio composto. Foram detectados e identificados os fungos associados às sementes em função das condições de luz e escuro, do tipo de placa utilizada (vidro e plástico) e do substrato empregado (agar-água e papel de filtro). Os critérios para a comparação destes métodos foram: sensibilidade, economicidade e praticidade.

O delineamento experimental foi aleatorizado em esquema fatorial $2 \times 2 \times 2$. Cada tratamento constou de quatro repetições de 50 sementes. Foram realizadas análises de variância dos dados obtidos, para verificar possíveis interações entre os fatores analisados (regime de luz, recipiente e substrato).

A ilustração dos tratamentos encontra-se na Figura 1.

Para levantamento dos fungos associados (gêneros/ espécies de fungos detectados) e incidência, realizou-se análise sanitária de sementes dos cruzamentos, empregando o método de placas de Petri de plástico contendo como substrato papel de filtro umedecido com água destilada. A incubação das sementes foi feita sob regime de luz alternada (12h luz branca fluorescente $/ 12 \mathrm{~h}$ escuro). As sementes foram incubadas por sete dias a $28 \pm 2{ }^{\circ} \mathrm{C}$.

Foram utilizadas sementes de 29 cruzamentos (biparentais e policruzamentos) realizados em 2002, 2003 e 2004, em condição ambiente e por isso havendo variações de um ano para outro ou um local para o outro, dentro da mesma estação experimental.

As avaliações foram realizadas examinando-se todas as sementes sob estereomicroscópio e, se necessário, microscópio composto. A identificação dos gêneros e espécies foi baseada em literatura especializada $(1,3,5,9)$.

Para verificar a relação entre a incidência fúngica e o ambiente onde foram produzidas as sementes de cana-de-açúcar, as incidências da análise sanitária foram relacionadas com dados climáticos coletados pelo CTC da região de produção das sementes dos cruzamentos considerando que a incidência fúngica pode ser afetada pela variação das temperatura $(\mathrm{T})$ e umidade relativa (UR) prevalente. Plotaram-se, em um gráfico, os dados de temperatura e umidade relativa em que cada cruzamento foi produzido. A temperatura e a umidade relativa foram calculadas por meio de médias das máximas e mínimas que ocorreram durante 20 dias (período aproximado desde o dia do cruzamento, até coleta das sementes). Os cruzamentos formados em diferentes condições climáticas foram divididos em grupos e, posteriormente, foi analisado o efeito do ambiente na incidência de cada fungo nas sementes de cana-de-açúcar.

Todos os dados obtidos foram transformados em arco sen $\sqrt{x / 100}$ e submetidos à análise de variância e as médias comparadas pelo Teste de Tukey, ao nível de $5 \%$ de probabilidade.

\section{RESULTADOS E DISCUSSÃO}

Os fungos detectados foram: Alternaria alternata; Aspergillus sp.; Bipolaris sacchari; três espécies agrupadas em grupos morfológicos (GM) distintos pertencentes ao gênero Bipolaris; dois grupos morfológicos de Cladosporium; Colletotrichum sp.; três grupos morfológicos de Curvularia; Epicoccum sp.; Fusarium verticillioides; Fusarium semitectum; Leptosphaerulina sp.; Nigrospora sp.; Penicillium sp.; Periconia sp.; Phoma herbarum; Rhizopus sp. e Trichoderma sp. Os mais freqüentemente encontrados foram: Bipolaris sacchari; Bipolaris spp.; Cladosporium spp.; Curvularia spp.; Fusarium verticillioides; Fusarium semitectum e Phoma herbarum. As fotos desses fungos estão apresentadas na Figura 2. Dentre os fungos encontrados, apenas os Curvularia GM1, Bipolaris sacchari, Cladosporium GM1, Fusarium verticillioides, Fusarium semitectum e Nigrospora sp. tiveram incidência, estatisticamente relevante, para permitir comparação entre tratamentos.

Os resultados da análise de variância (Tabelas 1, 2 e 3) mostraram que para Cladosporium GM1, o regime de luz e o substrato interagiram positivamente, quando se utilizou papel de filtro e luz alternada.

Essa mesma análise indicou que, para o crescimento de Bipolaris sacchari, o regime de luz teve influência em todos os cruzamentos avaliados, desenvolvendo-se melhor em condições de luz alternada. No cruzamento SP953387 x RB815396 houve, também, significância na interação entre regime de luz e substrato, com maior incidência fúngica nas sementes submetidas à combinação papel de filtro e luz alternada .

Com relação a Curvularia GM1, o resultado das interações variou de acordo com o cruzamento. No cruzamento SP81231 X SP963295 houve interação entre regime de luz e substrato, e entre recipiente e substrato. A melhor combinação foi papel de filtro, luz alternada e placa de plástico. Quanto ao cruzamento SP992254 X SP953163, apenas a interação entre regime de luz e recipiente foi significativa. Curvularia GM1 apresentou maiores incidências quando as sementes foram incubadas em placa de Petri de vidro e luz alternada, ou placa de 


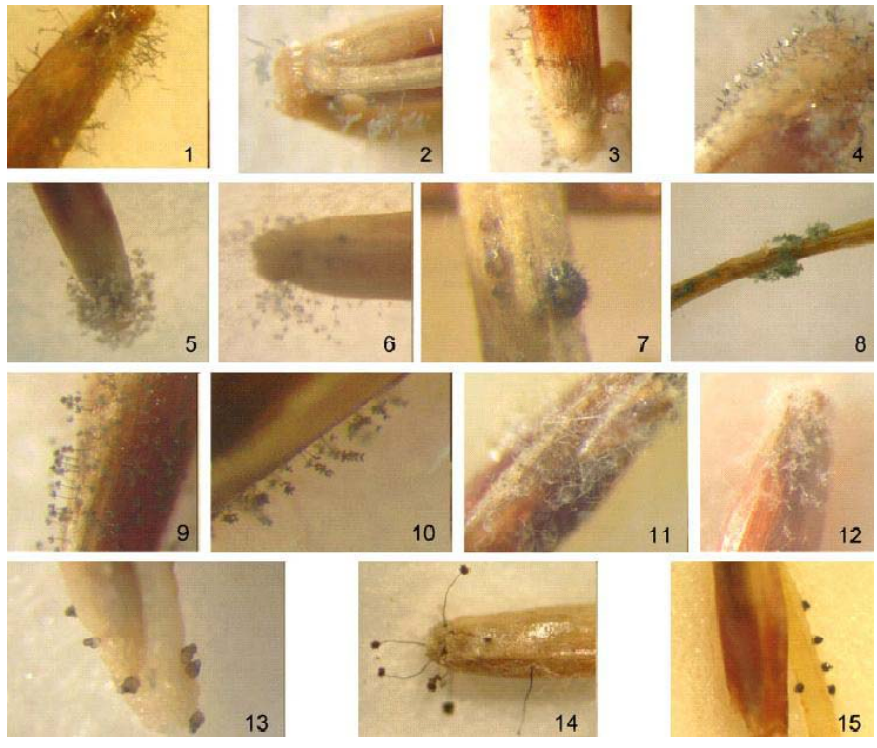

Figura 2 - Fungos encontrados nas análises sanitárias: Alternaria alternata (1), Penicillium sp. (2), Bipolaris sacchari (3), Bipolaris GM1 (4), Cladosporium GM1 (5), Cladosporium GM2 (6), Colletotrichum sp. (7), Trichoderma sp. (8), Curvularia GM1 (9), Curvularia GM3 (10), Fusarium verticillioides (11), Fusarium semitectum (12), Leptosphaerulina sp. (13), Periconia sp. (14), Phoma herbarum (15).

Petri de plástico e escuro contínuo. No cruzamento SP953387 X RB815396, houve diferença significativa apenas entre regimes de luz, observando-se as maiores incidências em luz alternada.

Entre as variáveis estudadas, no cruzamento SP953387 X
RB815396, não houve interação significativa para Fusarium verticillioides. No cruzamento SP992254 X SP953163, houve interação entre recipiente e regime de luz (sendo melhor a placa de Petri de vidro e escuro) e interação substrato e regime de luz (sendo melhores: agar-água e escuro, papel de filtro e luz alternada). Esses dados indicam, para Fusarium verticillioides, que a interação entre escuro, agar-água e vidro é a que propicia maior recuperação do fungo.

Fusarium semitectum não apresentou diferenças de incidência para as diferentes variáveis comparadas, com exceção do substrato papel de filtro, que permitiu aumento significativo na incidência desse fungo.

Os dados mostraram também diferenças significativas de incidência de Nigrospora sp. na interação regime de luz e substrato. As maiores incidências ocorreram na interação luz alternada e agarágua.

Para Aspergillus sp. houve interação significativa entre regime de luz e substrato e entre recipiente e substrato, sendo papel de filtro e placa de plástico e luz e papel de filtro respectivamente as melhores interações.

Dentre os métodos comparados, verificou-se que para Bipolaris sacchari, Curvularia GM1, Aspergillus sp., o melhor foi regime de luz alternada, placa de Petri de plástico e papel de filtro. Esse resultado era esperado, pois se sabia que a maioria dos fungos requer luz alternada para sua esporulação e que o papel de filtro mantém a umidade necessária para seu crescimento. Para o gênero Fusarium que, de acordo com Booth (3), deveria se desenvolver em regime de luz alternada, foi observado o contrário. Fusarium semitectum desenvolveu-se igualmente tanto na presença quanto

Tabela 1. Significância das interações metodológicas entre regime de luz, recipiente e substrato, na incidência fúngica em sanidade de sementes do cruzamento SP81231 X SP963295

\begin{tabular}{|c|c|c|c|c|}
\hline \multirow[b]{2}{*}{ fatores } & \multicolumn{4}{|c|}{ CRUZAMENTO SP81231 X SP963295 } \\
\hline & Cladosporium sp. & Bipolaris sacchari & $\begin{array}{l}\text { Curvularia sp. } 1 \\
\text { (\%)a }(\%)\end{array}$ & Nigrospora $s p$ \\
\hline Regime de luz & $*$ & $*$ & n.s. & $*$ \\
\hline Recipiente & n.s. & n.s. & n.s. & n.s. \\
\hline Substrato & * & n.s. & * & * \\
\hline Regime de luz X recipiente & n.s. & n.s. & n.s. & n.s. \\
\hline Regime de luz X substrato & * & n.s. & * & n.s. \\
\hline Recipiente X substrato & n.s. & n.s. & * & * \\
\hline Regime de luz X recipiente Xsubstrato & n.s. & n.s. & n.s. & n.s. \\
\hline $\mathrm{CV}(\%)$ & 8 & 27 & 24 & 39 \\
\hline
\end{tabular}

Tabela 2 - Significância das interações metodológicas entre regime de luz, recipiente e substrato, na incidência fúngica em sanidade de sementes do cruzamento SP992254 X SP953163

\begin{tabular}{lccc}
\hline & \multicolumn{2}{c}{ CRUZAMENTO SP992254 X SP953163 } \\
fatores & Fusarium verticillioides & Bipolaris sacchari & Curvularia sp. 1 \\
\hline Regime de luz & $*$ & $*$ & n.s. \\
Recipiente & n.s & n.s & n.s. \\
Substrato & $*$ & n.s. & n.s. \\
Regime de luz X recipiente & $*$ & n.s. & $*$ \\
Regime de luz X substrato & $*$ & n.s. & n.s. \\
Recipiente X substrato & n.s. & n.s. & n.s. \\
Regime de luz X recipiente X substrato & n.s. & n.s. & n.s. \\
\hline CV $(\%)$ & 27 & 25 & 13 \\
\hline
\end{tabular}


Tabela 3 - Significância das interações metodológicas entre Regime de luz, recipiente e substrato, na incidência fúngica em sanidade de sementes do cruzamento SP953387 X RB815396

\begin{tabular}{|c|c|c|c|c|c|c|}
\hline \multirow[b]{2}{*}{ fatores } & \multicolumn{6}{|c|}{$\begin{array}{c}\text { CRUZAMENTO SP953387 X RB815396 } \\
\text { Incidência fúngica }(\%)\end{array}$} \\
\hline & Fusarium & verticillioides & Fusarium semitectum & Bipolaris sacchari & Curvularia sp. 1 & Aspergillus sp. \\
\hline Regime de luz & & n.s. & n.s. & $*$ & $*$ & * \\
\hline Recipiente & & n.s. & n.s. & n.s. & n.s. & n.s. \\
\hline Substrato & & n.s. & $*$ & n.s. & n.s. & $*$ \\
\hline Regime de luz X recipiente & & n.s. & n.s. & n.s. & n.s. & n.s. \\
\hline Regime de luz X substrato & & n.s. & n.s. & $*$ & n.s. & $*$ \\
\hline Recipiente X substrato & & n.s. & n.s. & n.s. & n.s. & $*$ \\
\hline Regime de luz $\mathrm{X}$ recipiente $\mathrm{X}$ substrato & & n.s. & n.s. & n.s. & n.s. & n.s. \\
\hline CV $(\%)$ & & 18 & 73 & 21 & 24 & 11 \\
\hline
\end{tabular}

na ausência de luz, porém o melhor substrato foi o papel de filtro. Já Fusarium verticillioides desenvolveu melhor na ausência de luz, no substrato agar-água em placas de Petri de vidro. Brasil (4), Mathur \& Kongsdal (9) e Lucca Filho (8) relataram que, tanto placas de Petri de vidro pírex como as de plástico transparente, permitem a mesma passagem de luz, assim, a diferença de material utilizado não influenciaria a incidência dos fungos. É importante salientar que, para Fusarium verticillioides, o emprego da placa de Petri de plástico proporcionou menor incidência do que o da placa de vidro. Possivelmente, algum outro fator, que não necessariamente a passagem da luz, influenciou neste resultado.

Levando-se em conta apenas o parâmetro sensibilidade, concluiuse que o melhor método, que mais se adequou para a maioria dos fungos patogênicos foi o método papel de filtro, regime de luz alternada e placa de Petri de plástico.

De acordo com o parâmetro praticidade, é importante salientar que todos aqueles tratamentos que exigem manufatura de meio de cultura requerem muito tempo de preparo para autoclavar e verter o meio. O uso de papel de filtro, como substrato, resultou em procedimeto mais simples e rápido.

Além desses parâmetros, o método do papel de filtro é mais econômico, não necessitando de gastos com agar-água, energia com autoclave e vidrarias no preparo. Pode ser realizado em quaisquer condições de laboratório, inclusive os menos equipados.

Finalmente, perante os dados apresentados, escolheu-se o método de placa de Petri de plástico, substrato papel de filtro e incubação sob regime de luz alternada ( $12 \mathrm{~h}$ luz branca fluorescente $/ 12 \mathrm{~h}$ escuro) para a realização dos levantamentos dos fungos associados e da incidência em sementes de cana-de-açúcar, realizados posteriormente.

Os resultados do teste de sanidade de sementes estão presentes na Tabela 4.

Os fungos encontrados nas sementes, no decorrer destes testes, foram os mesmos encontrados na fase anterior, de comparação entre métodos. Os gêneros mais freqüentes, nos 29 cruzamentos avaliados nesta fase, foram: Bipolaris, Cladosporium, Curvularia, Fusarium e Phoma. As espécies ou grupos morfológicos com freqüencia média superior a $5 \%$ foram Fusarium verticillioides, Bipolaris sacchari, Curvularia GM1, Phoma herbarum e Cladosporium GM1.

Observou-se semelhança entre os gêneros fúngicos associados às sementes de cana-de-açúcar encontrados neste estudo e aqueles relatados em pesquisas anteriores, como as relatadas por Sanguino (12), Byther \& Steiner (2), Loveless \& Smith (7) e Sinha \& Singh (14), como os gêneros: Bipolaris, Cladosporium, Curvularia, Alternaria, Aspergillus, Fusarium, Phoma, Penicillium, Rhizopus, Epicoccum e Nigrospora. Há semelhança também quanto a algumas espécies Bipolaris sacchari, Fusarium semitectum, já encontradas em trabalhos realizados anteriormente e neste.

No presente estudo não foram encontrados os gêneros Pestalotia, Monilia, Phytomices, anteriormente relatados no Brasil por Sanguino (12) e outros como Cephalosporium, Chaetomium, Diplodia e Lacelinopsis, relatados apenas na Índia por Narendra \& Setty (10).

Com relação a fungos nunca antes relatados em associação com sementes de cana-de-açúcar, no presente estudo, encontraram-se os gêneros Colletotrichum, Leptosphaerulina e Periconia, entretanto, em baixas incidências, não sendo considerados de grande importância atual. Há que ser comentado que existe flexibilidade quanto à importância de fungos, pois um organismo, em um determinado momento, sem grandes importâncias, no futuro, poderá ser um agente causal de doenças devastadoras. Por isso, estudos futuros, com esses novos patógenos devem ser desenvolvidos.

As incidências encontradas neste e em outras pesquisas foram comparadas e percebeu-se que existe certa variabilidade nos resultados. Cada um dos estudos mostra incidências diferentes, porém os gêneros que apresentaram maiores delas foram: Alternaria, Aspergillus, Chetomium, Curvularia e Bipolaris, na Índia (10, 14). No Brasil,

Tabela 4 - Incidências (\%) de fungos em sementes de cana-de-açúcar provenientes de 29 cruzamentos do programa de melhoramento genético de canade-açúcar do CTC nos anos de 2002, 2003 e 2004

\begin{tabular}{|c|c|c|c|c|c|c|c|c|c|c|c|c|c|}
\hline & & $\begin{array}{c}\text { Fusarium } \\
\text { verticillioides }\end{array}$ & $\begin{array}{l}\text { Fusarium } \\
\text { semitectum }\end{array}$ & $\begin{array}{c}\text { Bipolaris } \\
\text { sacchari }\end{array}$ & $\begin{array}{c}\text { Bipolaris } \\
\text { GM1 }\end{array}$ & $\begin{array}{c}\text { Bipolaris } \\
\text { GM2 }\end{array}$ & $\begin{array}{c}\text { Phoma } \\
\text { herbarum }\end{array}$ & $\begin{array}{l}\text { Cladosporium } \\
\text { GM1 }\end{array}$ & $\begin{array}{l}\text { Cladosporium } \\
\text { GM2 }\end{array}$ & $\begin{array}{c}\text { Curvularia } \\
\text { GM1 }\end{array}$ & $\begin{array}{c}\text { Curvularia } \\
\text { GM2 }\end{array}$ & $\begin{array}{c}\text { Curvularia } \\
\text { GM3 }\end{array}$ & $\begin{array}{l}\text { Penicillium } \\
\text { sp. }\end{array}$ \\
\hline 2002 & Média anual & 20 & 5 & 8 & 1 & 0 & 5 & 7 & 5 & 7 & 1 & 1 & 2 \\
\hline 2003 & Média anual & 18 & 4 & 12 & 2 & 1 & 12 & 10 & 5 & 11 & 1 & 0 & 1 \\
\hline \multirow[t]{2}{*}{2004} & Média anual & 12 & 2 & 4 & 1 & 2 & 5 & 13 & 5 & 17 & 0 & 0 & 0 \\
\hline & Média geral 17 & 4 & 10 & 1 & 1 & 9 & 10 & 5 & 10 & 1 & 0 & 1 & \\
\hline
\end{tabular}


Tabela 5 - Incidência de fungos em sementes de cana-de-açúcar provenientes de 29 cruzamentos produzidos em diferentes faixas de ambiente

\begin{tabular}{|c|c|c|c|c|c|c|c|c|}
\hline \multirow[b]{2}{*}{$\begin{array}{l}\text { Grupo } \\
\text { Climático }\end{array}$} & \multirow[b]{2}{*}{$\begin{array}{l}\text { Tmédia } \\
\left({ }^{\circ} \mathrm{C}\right)\end{array}$} & \multirow[b]{2}{*}{$\begin{array}{l}\text { UR } \\
(\%)\end{array}$} & \multicolumn{6}{|c|}{ Incidência média $(\%)$} \\
\hline & & & $\begin{array}{c}\text { Fusarium } \\
\text { verticillioides }\end{array}$ & $\begin{array}{c}\text { Fusarium } \\
\text { semitectum }\end{array}$ & $\begin{array}{l}\text { Bipolaris } \\
\text { sacchari }\end{array}$ & $\begin{array}{l}\text { Bipolaris } \\
\text { GM1 }\end{array}$ & $\begin{array}{l}\text { Bipolaris } \\
\text { GM2 }\end{array}$ & $\begin{array}{c}\text { Phoma } \\
\text { herbarum }\end{array}$ \\
\hline $\mathrm{GC} 1$ & $26,5-29$ & $80-90$ & $20,2 \mathrm{a} *$ & $4,6 \mathrm{a}$ & $8,4 \mathrm{a}$ & $0,7 \mathrm{a}$ & $0,4 \mathrm{a}$ & $5,1 \mathrm{a}$ \\
\hline $\mathrm{GC} 2$ & $25-26$ & $92-97$ & $17 \mathrm{a}$ & $3,8 \mathrm{a}$ & $13 a$ & $1,6 \mathrm{a}$ & $0,5 \mathrm{a}$ & $12,8 \mathrm{a}$ \\
\hline $\mathrm{GC} 3$ & $26,5-28,5$ & $98-100$ & $11,6 \mathrm{a}$ & $2 \mathrm{a}$ & $3,6 \mathrm{a}$ & $1 \mathrm{a}$ & $1,6 \mathrm{a}$ & $5,3 \mathrm{a}$ \\
\hline Média total & - & - & 16 & 3 & 8 & 1 & 1 & 8 \\
\hline \multirow[b]{2}{*}{$\begin{array}{l}\text { Grupo } \\
\text { Climático }\end{array}$} & \multirow[b]{2}{*}{$\begin{array}{l}\text { Tmédia } \\
\left({ }^{\circ} \mathrm{C}\right)\end{array}$} & \multirow[b]{2}{*}{$\begin{array}{l}\text { UR } \\
(\%)\end{array}$} & \multicolumn{6}{|c|}{ Incidência média (\%) } \\
\hline & & & $\begin{array}{c}\text { Cladosporium } \\
\text { GM1 }\end{array}$ & $\begin{array}{c}\text { Cladosporium } \\
\text { GM2 }\end{array}$ & $\begin{array}{c}\text { Curvularia } \\
\text { GM1 }\end{array}$ & $\begin{array}{c}\text { Curvularia } \\
\text { GM2 }\end{array}$ & $\begin{array}{c}\text { Curvularia } \\
\text { GM3 }\end{array}$ & $\begin{array}{c}\text { Penicillium } \\
\text { sp. }\end{array}$ \\
\hline GC1 & $26,5-29$ & $80-90$ & $7 a$ & $5 \mathrm{a}$ & $7,2 \mathrm{a}$ & $0,7 \mathrm{a}$ & $0,6 \mathrm{a}$ & $1,6 \mathrm{a}$ \\
\hline $\mathrm{GC} 2$ & $25-26$ & $92-97$ & $11 \mathrm{a}$ & $5 \mathrm{a}$ & $11 \mathrm{a}$ & $0,6 \mathrm{a}$ & $0,33 \mathrm{a}$ & $0,73 \mathrm{a}$ \\
\hline $\mathrm{GC} 3$ & $26,5-28,5$ & $98-100$ & $13 \mathrm{a}$ & $5 \mathrm{a}$ & $16,6 \mathrm{a}$ & $0 \mathrm{a}$ & $0 \mathrm{a}$ & $0,33 \mathrm{a}$ \\
\hline Média total & - & - & 10 & 5 & 12 & 0 & 0 & 1 \\
\hline
\end{tabular}

* Médias seguidas por letras iguais nas colunas, não diferem estatisticamente entre si pelo teste de Tukey ao nível de $5 \%$ de probabilidade.

$\mathrm{GC}=$ grupo climático.

aqueles mais encontrados foram Curvularia, Bipolaris, Phoma e Fusarium (12). Este dado é concordante ao encontrado no presente estudo.

Quanto à relação entre incidência fúngica nas sementes e o ambiente onde foram produzidas, os resultados estão apresentados na Tabela 5.

Pela tabela, verifica-se que a hipótese de que a incidência fúngica varia com a temperatura (T) e a umidade relativa (UR) na formação das sementes não foi comprovada. Essa afirmação se baseia nos resultados de que as incidências fúngicas não diferiram estatisticamente entre os grupos climáticos formados. As variações de incidências podem estar associadas a outros fatores como fonte de inóculo ou características genéticas das sementes. Talvez essa hipótese possa ser verdadeira com faixas de clima mais espaçadas, diferentemente das ocorridas nos anos de formação das sementes usadas neste experimento, onde a faixa foi pequena.

O método considerado mais adequado para a detecção de fungos associados às sementes de cana-de-açúcar foi placa de Petri de plástico, substrato papel de filtro e incubação por sete dias sob regime de luz alternada (12h luz branca fluorescente/ $12 \mathrm{~h}$ escuro). Tal método foi o mais econômico, sensível e prático.

Os gêneros fúngicos mais comuns encontrados em sementes de cana-de-açúcar foram Bipolaris, Cladosporium, Curvularia, Fusarium e Phoma, fungos estes, presentes na maioria dos cruzamentos e que apresentaram maiores incidências. Quanto a esses fungos não se sabe a patogenicidade na fase de plântula, no entanto, sabe-se que na canade-açúcar adulta fungos como Bipolaris, Curvularia e Fusarium são agentes causais de doenças e possivelmente poderão causar problemas em sementeira. Phoma e Cladosporium, mesmo não sendo importantes patógenos em cana-de-açúcar adulta, não se sabe o efeito em plântulas. Sobre esses fungos, portanto, devem ser feitos novos estudos a respeito da patogenicidade para se estabelecer o foco dos tratamentos químicos e assim diminuir a ocorrência de doenças e morte de plântulas em cruzamentos promissores.

Não foi constatada relação de temperatura e umidade relativa na formação das sementes de cana-de-açúcar com incidência fúngica nas situações do presente trabalho. Supõe-se que as variações possam estar relacionadas com diferentes fontes de inóculo nos locais de cruzamento ou características genéticas das sementes.

\section{REFERÊNCIAS BIBLIOGRAFICAS}

1. Barnett, H.L.; Hunter, B.B. Illustrated genera of imperfect fungi. 4th. ed. Saint Paul: The American Phytopathological Society, 1998. $218 \mathrm{p}$.

2. Byther, R.S.; Steiner, G.W. Four sugarcane seedling diseases in Hawaii: causal agents, control, and a selective medium for isolation. Phytopathology, Saint Paul, v. 62, p. 120-124, 1972.

3. Booth, C. The genus fusarium. Kew: CAB, 1971. $237 \mathrm{p}$.

4. Brasil. Ministério da Agricultura e da Reforma Agrária. Regras para análise de sementes. Brasília: SNDA; DNDV; CLAV, 1992. 206 p.

5. Hanlin, R.T. Illustrated genera of ascomycetes. Minnesota: The American Phytopathological Society, 1990. 263 p.

6. Heinz, D.J. (Ed.). Sugarcane improvement through breeding. Amsterdam: Elsevier Science Publishers B.V., 1987. 603 p.

7. Loveless, A.R.; Smith, C.E.M. Seedling blight of sugar-cane: a new disease caused by Helminthosporium sacchari Butler. Annals of Applied Biology, Warwick, v. 44, n. 4, p. 419-424, 1956.

8. Lucca Filho, O. Metodologia dos testes de sanidade de sementes. In: Soave, J.; Wetzel, M.M.V.S. (Ed.). Patologia de sementes. Campinas: Fundação Cargill, 1987. p. 430-440.

9. Mathur, S.B.; Kongsdal, O. Common laboratory seed health testing methods for detecting fungi. Basserdorf: International Seed Testing Association, 2003. 425 p.

10. Narendra, D.V.; Setty, M.V.N. Seed mycoflora of sugarcane and their importance in nurseries. Seed Research, New Delhi, v. 7, n. 2, p. 145-150, 1979.

11. Rego, A.M. Análise sanitária na produção de sementes de hortaliças. In: Zambolim, L., (Ed.). Sementes: qualidade fitossanitária. Viçosa: Universidade Federal de Viçosa; Departamento de Fitopatologia, 2005. p. 267-294.

12. Sanguino, A. Patologia e controle dos fungos de sementes de cana-de-açúcar e resistência de progênies à Helminthosporium sacchari. 1976. 76 p. Dissertação (Mestrado em Fitopatologia) - Escola Superior de Agricultura "Luiz de Queiroz", Universidade de São Paulo, Piracicaba.

13. Silva, W.M. Pré-seleção de "seedlings" de cana-de-açúcar resistentes a Ustilago scitaminea, pela inoculação das "sementes". 1978. 65 p. Tese (Doutorado em Fitopatologia) - Escola Superior de Agricultura "Luiz de Queiroz", Universidade de São Paulo, Piracicaba, 1978.

14. Sinha, O.K.; Singh, K. Sugarcane seed decay: prevalence of fungi and collar rot of seedlings caused by Curvularia spp. and Drechslera spp. Seed Research, New Delhi, v. 10, n. 2, p. 114-119, 1982. 